\title{
Isoflavone Metabolites Cis- and Trans-Tetrahydrodaidzein Improve Plasma Lipid Profile in Apolipoprotein(E)-Deficient Mice
}

\author{
Fan Jiang ${ }^{1,2, *}$, Alan J. Husband ${ }^{3,4}$ and Gregory J. Dusting ${ }^{1,2}$ \\ ${ }^{1}$ Howard Florey Institute, University of Melbourne, Victoria 3010, Australia \\ ${ }^{2}$ Bernard O'Brien Institute of Microsurgery, University of Melbourne, Victoria, Australia \\ ${ }^{3}$ Novogen Ltd, 140 Wicks Road, North Ryde, NSW 2113, Australia \\ ${ }^{4}$ Faculty of Veterinary Science, The University of Sydney, NSW, 2006, Australia
}

\begin{abstract}
Isoflavones have been suggested to have hypolipidemic effects. As these compounds undergo complex metabolism in the body, the biological actions of isoflavone metabolites are largely unknown. We studied the hypolipidemic effects of two isoflavone metabolites initially identified in the human urine, cis-tetrahydrodaidzein (NV-04) and transtetrahydrodaidzein (NV-05) in apolipoprotein(E)-deficient mice fed with a high-fat diet. NV-04 and NV-05 were mixed in the food (200 mg per kg) and the treatment lasted for 16 weeks. NV-04 decreased plasma levels of LDL by 30\%, triglycerides by $65 \%$ and increased HDL/LDL ratio by $70 \%$. These effects of NV-04 are specific since its stereoisomer NV-05 is less effective. Notably, the effects of NV-04 are observed in the absence of apolipoprotein(E), therefore our results suggest that using cis-tetrahydrodaidzein as a complimentary medicine in hyperlipidemic patients with apolipoprotein(E) dysfunctions warrants further investigations.
\end{abstract}

Keywords: Isoflavone, metabolite, hyperlipidemia, apolipoprotein(E), LDL, HDL.

\section{INTRODUCTION}

Hyperlipidemia is a well-established risk factor for coronary arterial disease (CAD). Correction of this pathophysiological status is one of the most important clinical goals in the management of patients with CAD. Apart from pharmacological treatment, dietary intervention is the primary therapeutic approach for persons who are at increased risk of $\mathrm{CAD}$ as a result of elevated cholesterol levels. Epidemiological data have suggested that ingestion of foods that are rich in soy protein is associated with reduced risk for CAD [1]. On the basis of such evidence, in 1999 the US Food and Drug Administration approved a health claim for the relationship between consumption of soy protein and reduced risk of coronary heart disease.

Soy proteins contain high levels of isoflavones. Although some animal and human studies have provided evidence that the isoflavone components may contribute to the observed hypolipidemic effect of soy protein, it is still controversial whether purified isoflavones have equivalent efficacy in blood cholesterol lowering to intact soy proteins [2]. Most of previous studies in this direction have focused on naturally occurring isoflavone molecules. However, phenolic compounds may undergo extensive metabolism after ingestion either by the gut microflora before their absorption into the intestinal mucosa or by cells of the intestine or liver after

*Address correspondence to this author at the Bernard O'Brien Institute of Microsurgery, 42 Fitzroy Street, Fitzroy, Victoria 3065, Australia; Tel: +613-9288 4044; Fax: +61-3-9416 0926; E-mail: fjiang @unimelb.edu.au absorption. The metabolism of polyphenols has a critical role in determining the overall bioavailability, and thus their biological effects, of each individual molecule. For example, the likely cardiovascular protective properties of red wine are thought to be attributable to the high content of procyanidins in the wine. However, animal studies demonstrated that native procyanidins were poorly absorbed by the intestine, whereas their microbial metabolites (low molecular weight phenolic acids) were readily detected in the urine, suggesting an extensive degradation of procyanidins by the gut microflora before absorption [3]. Therefore, it is speculated that the observed effects of procyanidin ingestion are, at least partly, attributable to their metabolites. The biological effects of these phenolic metabolites remain largely unknown. Emerging evidence has shown that polyphenol metabolites may have higher biological activities than the primary compounds. For example, the metabolites of rutin and quercetin, 3,4-dihydroxyphenylacetic and 4-hydroxyphenylacetic acids have greater inhibitory effects on platelet aggregation than their parent compounds. In addition, there is evidence that equol, a metabolite of the isoflavone daidzein, has a higher affinity to estrogen receptors than daidzein itself (reviewed in [4]). These results indicate that exploration of the biological activity of isoflavone metabolites may advance our understanding of the mechanisms of the proposed cardiovascular protective effects of isoflavones.

Our previous studies have shown that two isoflavone metabolites, dihydrodaidzein and dehydroequol, lowered total cholesterol and low density lipoprotein (LDL), but also lowered high density lipoprotein (HDL) in apolipoprotein(E)- 


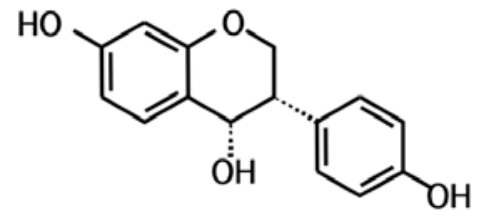

Cis-tetrahydrodaidzein

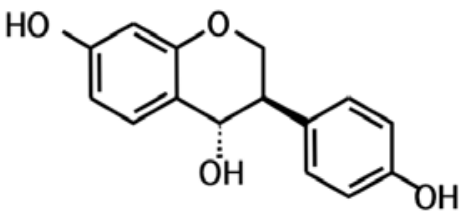

Trans-tetrahydrodaidzein

Fig. (1). Chemical structures of cis-tetrahydrodaidzein (NV-04) and trans-tetrahydrodaidzein (NV-05).

deficient $\left(a p o E^{0}\right)$ mice fed a high-fat diet [5]. As HDL is protective, an ideal isoflavone supplement is expected to lower the total cholesterol and LDL levels without compromising the HDL level.

Cis-tetrahydrodaidzein (NV-04) and trans-tetrahydrodaidzein (NV-05) are other isoflavone metabolites that were initially identified in human urine [6, 7] (see Fig. 1 for their structures). In previous studies, it was shown that NV-04 and/or NV-05 had multiple vascular protective effects, including endothelium-dependent vasodilatation, inhibition of smooth muscle proliferation, and suppression of balloon injury induced arterial neointima formation [8-10]. In the present study we report that NV-04 improved the plasma lipoprotein profile in an apolipoprotein(E)-independent manner in mice.

\section{MATERIALS AND METHODS}

\section{Reagents}

The following reagents were used: ammonium chloride; L-glutamic dehydrogenase; $\alpha$-ketoglutarate; $N$-(1-naphthyl) ethylenediamine dihydrochloride; $\beta$-nicotinamide adenine dinucleotide phosphate ( $\beta$-NADPH); nitrate reductase; sulfanilamide; 1,1,3,3-tetramethoxypropane; thiobarbituric acid. All of these reagents were from Sigma-Aldrich.

Tetrahydrodaidzein isomers used in this study were synthesized by sodium borohydride $(\mathrm{NaBH} 4)$ reduction method as described previously [11] and were provided by Novogen Ltd (Sydney, Australia).

\section{Animals and Treatment Protocol}

This study is in compliance with the guidelines of Animal Experimentation Ethics Committee of the Howard Florey Institute and National Health and Medical Research Council of Australia. Male apoE $\mathrm{E}^{0}$ mice (with greater than 99\% C57BL/6 background) were purchased from the Animal Resource Centre (Western Australia) when they were 4 - 5 weeks old. Animals were randomly assigned to either a cholesterol $(0.15 \%)$-enriched high-fat diet with $42 \%$ of total energy being derived from milk fat (Harlan Teklad, Madison, Wisconsin, USA) (control group), or the same high-fat diet supplemented with $200 \mathrm{mg}$ per $\mathrm{kg}$ cis-tetrahydrodaidzein (NV-04 group) or trans-tetrahydrodaidzein (NV-05 group). Animals had free access to food and water.

After 16 weeks of treatment, animals were anaesthetized with ketamine (100 $\left.\mathrm{mg} \mathrm{kg}^{-1}\right)$ plus xylazine $\left(12 \mathrm{mg} \mathrm{kg}^{-1}\right.$, ip.), heparinized (3000 $\mathrm{U} \mathrm{kg}^{-1}$, ip.) and killed by carotid bleeding. Blood samples were collected into chilled microcentrifuge tubes, hearts and testicles were removed into cold phosphatebuffered saline (PBS).

\section{Plasma Lipid Measurement}

Blood samples were centrifuged at $15,000 \mathrm{~g}$ for $5 \mathrm{~min}$. The plasma was removed into a new $1.5 \mathrm{ml}$ vial, snap frozen in liquid nitrogen and stored at $-80^{\circ} \mathrm{C}$. Plasma total cholesterol, LDL cholesterol, HDL cholesterol and triglycerides were measured with a Synchron Cx Clinical System (Beckman) using reagent kits from Genzyme Co. (MA, USA) (for LDL) or Beckman Instruments Inc. (CA, USA) (for other lipids).

\section{Thiobarbituric Acid-Reactive Substances (TBARS) As- say}

The total oxidative stress was assessed by measuring the lipid peroxidation product malondialdehyde in the plasma using a colorimetric method based on that described by Satoh [12]. TBARS reagent was prepared by mixing 1:1 of $20 \%$ trichloroacetic acid with $1 \%$ thiobarbituric acid. Aliquots of $20 \mu \mathrm{l}$ plasma samples were diluted in $80 \mu \mathrm{l}$ distilled $\mathrm{H}_{2} \mathrm{O}$. The sample was mixed with $400 \mu \mathrm{l}$ TBARS reagent, heated to $95^{\circ} \mathrm{C}$ for $20 \mathrm{~min}$, and then cooled to room temperature. The colored reaction product was extracted with $500 \mu \mathrm{l}$ $n$-butanol by vigorous shaking for $20 \mathrm{~min}$, and the organic phase was separated from the mixture by centrifugation at $5000 \mathrm{~g}$ for $5 \mathrm{~min}$. A volume of $300 \mu \mathrm{l}$ of the supernatant was loaded into a 96-well plate and the absorbance at $550 \mathrm{~nm}$ (A550) was determined with a microplate reader (Molecular Devices). Serial dilutions of 1,1,3,3-tetramethoxypropane [malonaldehyde bis(dimethyl acetal)] were used to construct the standard curve.

\section{Plasma Nitrite/Nitrate Measurement}

The total plasma nitrate plus nitrite concentration was determined by Griess method [13]. Briefly, $25 \mu 1$ of plasma sample was diluted in $75 \mu \mathrm{l}$ PBS, deproteinized by centrifugation at $12,000 \mathrm{~g}$ for $40 \mathrm{~min}$ at $4^{\circ} \mathrm{C}$ using Vivaspin500 filter units with a 10,000 Molecular Weight Cutoff (Vivascience AG). The samples were loaded into a 96-well plate. Nitrate in the sample was converted to nitrite by incubation of the sample with $0.2 \mathrm{mM} \beta-\mathrm{NADPH}$ and $14 \mathrm{mU}$ nitrate reductase in PBS in a total volume of $200 \mu \mathrm{l}$ at room temperature for 3 hr. Then to each well, $50 \mu \mathrm{GDH}$ reaction mix, containing 2 $\mathrm{mg} \mathrm{ml^{-1 }} \alpha$-ketoglutarate, $1.5 \mathrm{U} \mathrm{ml}^{-1}$ L-glutamic dehydrogenase (GDH) and $0.3 \mathrm{M}$ ammonium chloride in PBS, was added and incubated at $37^{\circ} \mathrm{C}$ for $30 \mathrm{~min}$. After reaction, 50 $\mu 1$ of Griess reagent A ( $1 \%$ sulfanilamide) and $50 \mu \mathrm{l}$ reagent B [0.1\% N-(1-naphthyl)ethylenediamine dihydrochloride] were added to each well and incubated at room temperature 
Table 1. Body, Heart and Testicle Weight Characteristics of Animals from all Groups

\begin{tabular}{|c|c|c|c|}
\hline & Control & NV04 & NV05 \\
\hline \hline Body weight (g) & $31.8 \pm 0.8(16)$ & $30.3 \pm 0.6(16)$ & $31.9 \pm 0.7(16)$ \\
\hline Heart weight (g) & $0.14 \pm 0.006(16)$ & $0.14 \pm 0.003(16)$ & $0.14 \pm 0.004(16)$ \\
\hline Testicle weight (g) & $0.18 \pm 0.003(15)$ & $0.17 \pm 0.004(16)$ & $0.17 \pm 0.006(16)$ \\
\hline Heart:body weight ratio (\%) & $0.45 \pm 0.01(16)$ & $0.47 \pm 0.01(16)$ & $0.45 \pm 0.02(16)$ \\
\hline Testicle:body weight ratio (\%) & $0.58 \pm 0.02(15)$ & $0.57 \pm 0.02(16)$ & $0.53 \pm 0.02(16)$ \\
\hline
\end{tabular}

Data are presented as mean \pm SE. Numbers in brackets are $n$.

for $10 \mathrm{~min}$ in dark. The Griess reaction product concentration was determined by A550, with serial dilutions of sodium nitrate being used as standards.

\section{Data Analysis}

Data are presented as mean \pm standard error $(\mathrm{SE})$. The mean data are analysed with one-way analysis of variance (one-way ANOVA) followed by Newman-Keuls test (for multiple comparisons). A value of $P<0.05$ was regarded as statistically significant.

\section{RESULTS AND DISCUSSION}

Chronic treatment with NV04 or NV05 as dietary supplementation for 16 weeks did not affect the growth rate of animals as demonstrated by the similar body weight in three groups (Table 1). To examine any possible estrogenic effect of these isoflavone metabolites on male reproductive organs, we also studied the testicle weight and the testicle to body weight ratio and found that these parameters were not affected by NV04 or NV05 treatment (Table 1), suggesting that NV04 and NV05 did not produce observable hypertrophy/atrophy of post-puberty testicles. Moreover, we found that treatment with NV04 or NV05 had little effect on heart weight or the heart to body weight ratio (Table $\mathbf{1}$ ).

In our previous study, we demonstrated that $\mathrm{apoE}^{0}$ mice fed with the high-fat diet developed severe hyperlipidemia as compared to control $\mathrm{C} 57 \mathrm{Bl} / 6$ mice fed with normal chow, with about 10-fold increase in the total cholesterol and about 4-fold increase in triglycerides [5]. ApoE is a constituent apolipoprotein in both chylomicrons and very low density lipoproteins (VLDL) and their remnant particles. ApoE binds to LDL receptor and LDL receptor-related protein (LRP) and mediates receptor-dependent clearance of atherogenic chylomicron remnant and VLDL remnant particles in the liver [14]. Therefore, the hyperlipidemia in $\mathrm{apoE}^{0}$ mice is largely resulted from remnant lipoprotein accumulation. The present study has shown that treatment with NV04 and NV05 reduced the plasma LDL cholesterol level by $30 \%$ and $19 \%$ respectively (both $P<0.05$ ), whereas the total and HDL cholesterol levels were not changed (Table 2). The mechanism(s) that mediates the LDL lowering effects of NV04 and NV05 is not clear. Borradaile and colleagues studied the effects of two primary soy isoflavones genistein and daidzein in a human hepatoma cell line (HepG2) in culture, and found that both compounds significantly increased LDL receptor expression accompanied by enhanced uptake and degrada- tion of LDL in these cells [15]. A functional role of LDL receptor in lipoprotein metabolism in vivo in $\mathrm{apoE}^{0}$ mice has been evidenced by apoE/LDL receptor double knockout mice, in which both VLDL and LDL levels were found higher than those in apo $\mathrm{E}^{0}$ mice [16]. In agreement with this notion, Murayama et al. have shown that overexpression of LDL receptors in the liver significantly reduced VLDL, VLDL remnant and LDL cholesterol levels in $\operatorname{apoE}^{0}$ mice [17], indicating that upregulation of LDL receptor expression can improve lipoprotein metabolism in apo $\mathrm{E}^{0}$ mice. Taking the evidence together, we postulate that regulation of LDL receptor expression may contribute to the observed hypolipidemic effects of NV04 and NV05, although a decreased VLDL secretion from the liver induced by these compounds can not be ruled out [15]. It should be noted that VLDL remnant metabolism in mice is different from that in human, for about $70 \%$ of the VLDL secreted from mouse liver contain apoB-48, but not apoB-100, whereas human liver only produces apoB-100 containing VLDL [18]. Since apoB-48 does not bind to LDL receptors, ie a large proportion of VLDL remnant in mice will not be affected by the LDL receptor availability, the potential effects of NV04 and NV05 on LDL cholesterol level in humans might be higher than those observed in mice. On the other hand, how apoB-48 containing chylomicron and VLDL remnants are metabolized in apoE ${ }^{0}$ mice is not clear.

NV04 markedly decreased the plasma triglyceride level by $65 \%(P<0.05)$, whereas the effect of NV05 is not significant (Table 2). Compelling evidence indicates that plasma triglyceride is an important factor in the progression of coronary artery disease [19]. This triglyceride lowering effect of NV04 can be explained by enhanced clearance of triglyceride-rich VLDL and its remnant through LDL receptors [17]. NV04 slightly increased the HDL level, although this effect is not significant. However, NV05 was totally void of such an effect. An interesting finding in this study is that NV04 remarkably elevated the HDL:LDL ratio by $72 \%$ as compared to the control group $(P<0.001)$, an effect that was not observed with NV05.

The beneficial effects of NV04 on plasma lipids appear to be specific since these effects are not produced by its stereoisomer NV05, and this difference in efficacy between the two tetrahydrodaidzein isoforms may be implicated in the negative findings with NV05 on plasma lipids in human beings [20]. Moreover, the hypolipidemic effects of NV04 are distinct from those observed in our previous study with 
Table 2. Effects of NV04 and NV05 on Plasma Lipids

\begin{tabular}{|c|c|c|c|}
\hline & Control & NV04 & NV05 \\
\hline \hline Total cholesterol (mM) & $22.7 \pm 1.3(16)$ & $23.0 \pm 1.6(16)$ & $20.2 \pm 1.1(16)$ \\
\hline LDL $(\mathbf{m M})$ & $16.6 \pm 0.85(16)$ & $11.6 \pm 0.88^{*}(16)$ & $13.4 \pm 0.72 *(16)$ \\
\hline HDL $(\mathbf{m M})$ & $4.2 \pm 0.23(16)$ & $4.73 \pm 0.27(15)$ & $3.69 \pm 0.19(16)$ \\
\hline Triglycerides (mM) & $4.95 \pm 0.46(12)$ & $1.74 \pm 0.34 *(14)$ & $3.31 \pm 0.55(16)$ \\
\hline HDL:LDL & $0.25 \pm 0.007(16)$ & $0.43 \pm 0.022 *(15)$ & $0.28 \pm 0.011(16)$ \\
\hline
\end{tabular}

Data are presented as mean \pm SE. Numbers in brackets are $n . * P<0.05 v s$ control, one-way ANOVA followed by Newman-Keuls test.

Table 3. Effects of NV04 and NV05 on Plasma Levels of TBARS and Nitrate/Nitrite (NOx)

\begin{tabular}{|c|c|c|c|}
\hline & Control & NV04 & NV05 \\
\hline \hline TBARS $(\mu M)$ & $16.0 \pm 0.46(16)$ & $17.7 \pm 0.69(15)$ & $14.4 \pm 0.75(16)$ \\
\hline NOx $(\mu M)$ & $73.0 \pm 4.45(16)$ & $70.0 \pm 4.60(16)$ & $72.8 \pm 3.95(16)$ \\
\hline
\end{tabular}

Data are presented as mean \pm SE. Numbers in brackets are $n$.

other two isoflavone metabolites dihydrodaidzein and dehydroequol, which nonspecifically reduced total, HDL and nonHDL cholesterol levels [5]. Importantly, the effects of NV04 are observed in the absence of apolipoprotein(E), therefore our results suggest that NV04 might be used as a complimentary medicine in hyperlipidemic patients with apolipoprotein(E) dysfunctions. Given that this compound is endogenously generated during normal isoflavone metabolism in the human body, it is likely that exogenous supplementation of NV04 is tolerable and safe.

Evidence has suggested that increased oxidative stress, particularly superoxide anion generation, and subsequent lipid peroxidation have critical roles in the development of atherosclerosis [21]. Excessive production of superoxide compromises endothelium-derived nitric oxide, which is a major endogenous protective molecule in the vasculature [22]. Our previous study showed that isoflavones have potent superoxide scavenging activities [5]. Therefore, in this study we examined whether NV04 and NV05 have any effects on systemic oxidative stress as assessed by the TBARS assay, and nitric oxide production as assessed by the total plasma nitrate/nitrite. However, the plasma TBARS level, a marker of lipid peroxidation [12], was not affected by NV04 or NV05 (Table 3). Likewise, the plasma levels of total nitrate/nitrite were not different amongst the three groups (Table 3). These results suggest that NV04 and NV05 do not have major effects in modulating lipid peroxidation and protecting nitric oxide function in vivo as suggested by other studies [23].

In summary, the isoflavone metabolite cis-tetrahydrodaidzein effectively decreased plasma LDL cholesterol and triglyceride levels and increased the HDL/LDL ratio in an apolipoprotein(E)-independent manner in mouse. Our results suggest that using cis-tetrahydrodaidzein as a complimentary medicine in hyperlipidemic patients with apolipoprotein(E) dysfunctions warrants further investigations.

\section{LIST OF ABBREVIATIONS}

$\begin{array}{lll}\mathrm{CAD} & = & \text { coronary arterial disease } \\ \mathrm{LDL} & = & \text { low density lipoprotein } \\ \mathrm{HDL} & = & \text { high density lipoprotein } \\ \operatorname{apoE}^{0} & = & \text { apolipoprotein(E)-deficient } \\ \mathrm{NV}-04 & = & \text { cis-tetrahydrodaidzein } \\ \mathrm{NV}-05 & = & \text { trans-tetrahydrodaidzein } \\ \mathrm{TBARS} & = & \text { thiobarbituric acid-reactive substances } \\ \mathrm{SE} & = & \text { standard error } \\ \text { ANOVA } & = & \text { analysis of variance } \\ \text { VLDL } & = & \text { very low density lipoproteins }\end{array}$

\section{CONFLICT OF INTEREST}

A.J.H is an employee of Novogen Ltd.

\section{ACKNOWLEDGMENTS}

This work was partly funded by Novogen Ltd, and partly by research grants from the National Health and Medical Research Council of Australia (983001, 217009). The authors thank Angela Gibson for assistance in the plasma lipid assays.

\section{REFERENCES}

[1] Goldberg AC. Perspectives on soy protein as a nonpharmacological approach for lowering cholesterol. J Nutr 1995; 125: 675S-678S.

[2] Demonty I, Lamarche B, Jones PJ. Role of isoflavones in the hypocholesterolemic effect of soy. Nutr Rev 2003; 61: 189-203.

[3] Gonthier MP, Donovan JL, Texier O, Felgines C, Remesy C, Scalbert A. Metabolism of dietary procyanidins in rats. Free Radic Biol Med 2003; 35: 837-44.

[4] Scalbert A, Morand C, Manach C, Remesy C. Absorption and metabolism of polyphenols in the gut and impact on health Biomed Pharmacother 2002; 56: 276-82. 
[5] Jiang F, Jones GT, Husband AJ, Dusting GJ. Cardiovascular protective effects of synthetic isoflavone derivatives in apolipoprotein e-deficient mice. J Vasc Res 2003; 40: 276-84.

[6] Kelly GE, Nelson C, Waring MA, Joannou GE, Reeder AY. Metabolites of dietary (soya) isoflavones in human urine. Clin Chim Acta 1993; 223: 9-22.

[7] Kim M, Kim SI, Han J, Wang XL, Song DG, Kim SU. Stereospecific biotransformation of dihydrodaidzein into (3S)equol by the human intestinal bacterium Eggerthella strain Julong 732. Appl Environ Microbiol 2009; 75: 3062-8.

[8] Chin-Dusting JP, Fisher LJ, Lewis TV, Piekarska A, Nestel PJ, Husband A. The vascular activity of some isoflavone metabolites: implications for a cardioprotective role. Br J Pharmacol 2001;133: 595-605.

[9] Ling S, Dai A, Williams MR, et al. The isoflavone metabolite cistetrahydrodaidzein inhibits ERK-1 activation and proliferation in human vascular smooth muscle cells. J Cardiovasc Pharmacol 2004; 43: 622-8.

[10] Kanellakis P, Nestel P, Bobik A. Angioplasty-induced superoxide anions and neointimal hyperplasia in the rabbit carotid artery: suppression by the isoflavone trans-tetrahydrodaidzein. Atherosclerosis 2004; 176: 63-72.

[11] Joannou GE, Kelly GE, Reeder AY, Waring M, Nelson C. A urinary profile study of dietary phytoestrogens. The identification and mode of metabolism of new isoflavonoids. J Steroid Biochem Mol Biol 1995; 54: 167-84.

[12] Satoh K. Serum lipid peroxide in cerebrovascular disorders determined by a new colorimetric method. Clin Chim Acta 1978; 90: $37-43$.

[13] Granger DL, Taintor RR, Boockvar KS, Hibbs JB, Jr. Measurement of nitrate and nitrite in biological samples using nitrate reductase and Griess reaction. Methods Enzymol 1996; 268: 142-51.
[14] Mahley RW, Ji ZS. Remnant lipoprotein metabolism: key pathways involving cell-surface heparan sulfate proteoglycans and apolipoprotein E. J Lipid Res 1999; 40: 1-16.

[15] Borradaile NM, de Dreu LE, Wilcox LJ, Edwards JY, Huff MW. Soya phytoestrogens, genistein and daidzein, decrease apolipoprotein B secretion from HepG2 cells through multiple mechanisms. Biochem J 2002; 366: 531-9.

[16] Ishibashi S, Herz J, Maeda N, Goldstein JL, Brown MS. The tworeceptor model of lipoprotein clearance: tests of the hypothesis in "knockout" mice lacking the low density lipoprotein receptor, apolipoprotein E, or both proteins. Proc Natl Acad Sci USA 1994; 91: 4431-5.

[17] Murayama T, Yokode M, Horiuchi H, Yoshida H, Sano H, Kita T. Overexpression of low density lipoprotein receptor eliminates apolipoprotein B100-containing lipoproteins from circulation and markedly prevents early atherogenesis in apolipoprotein E-deficient mice. Atherosclerosis 2000; 153: 295-302.

[18] Higuchi K, Kitagawa K, Kogishi K, Takeda T. Developmental and age-related changes in apolipoprotein B mRNA editing in mice. J Lipid Res 1992; 33: 1753-64.

[19] Hodis HN, Mack WJ. Triglyceride-rich lipoproteins and the progression of coronary artery disease. Curr Opin Lipidol 1995; 6 : 209-14.

[20] Nestel P, Fujii A, Zhang L. An isoflavone metabolite reduces arterial stiffness and blood pressure in overweight men and postmenopausal women. Atherosclerosis 2007; 192: 184-9.

[21] Stocker R, Keaney JF, Jr. Role of oxidative modifications in atherosclerosis. Physiol Rev 2004; 84: 1381-478.

[22] Dusting GJ, Macdonald PS. Endogenous nitric oxide in cardiovascular disease and transplantation. Ann Med 1995; 27: 395- 406.

[23] Di Giacomo C, Acquaviva R, Sorrenti V, et al. Oxidative and antioxidant status in plasma of runners: effect of oral supplementation with natural antioxidants. J Med Food 2009; 12: 145-50.

Received: May 12, 2009

Revised: July 16, 2009

Accepted: July 18, 2009

(C) Jiang et al.; Licensee Bentham Open.

This is an open access article licensed under the terms of the Creative Commons Attribution Non-Commercial License (http://creativecommons.org/licenses/by-nc/3.0/) which permits unrestricted, non-commercial use, distribution and reproduction in any medium, provided the work is properly cited. 